\title{
Some Properties of Generalized Frank Matrices
}

\author{
Efruz Özlem Mersin, Mustafa Bahşi* and Ayşe Dilek Maden
}

\begin{abstract}
In this paper, we first introduce a new generalization of Frank matrix which is a lower Hessenberg matrix. Then, we examine its algebraic structure, determinant, inverse, $L U$ decomposition and characteristic polynomial.
\end{abstract}

Keywords: Frank matrix; determinant; inverse; $L U$ decomposition; characteristic polynomial.

AMS Subject Classification (2020): Primary: 15B99 ; Secondary: 15A09; 15A15; 15A23.

*Corresponding author

\section{Introduction}

In 1958, Frank [3] defined an $n \times n$ matrix $F$ as

$$
F=\left[\begin{array}{cccccc}
n & n-1 & 0 & \cdots & 0 & 0 \\
n-1 & n-1 & n-2 & \cdots & 0 & 0 \\
n-2 & n-2 & n-2 & \cdots & 0 & 0 \\
\vdots & \vdots & \vdots & \ddots & \vdots & \vdots \\
2 & 2 & 2 & \cdots & 2 & 1 \\
1 & 1 & 1 & \cdots & 1 & 1
\end{array}\right]
$$

which is called Frank matrix $[4,7]$. One can easily generate the elements of matrix $F=\left[f_{i j}\right]$ by the rule:

$$
f_{i j}=\left\{\begin{array}{cc}
n+1-\max (i, j), & i>j-2 \\
0, & \text { otherwise. }
\end{array}\right.
$$

Frank matrix is one of the popular test matrices for eigenvalue routines, because the matrix $F$ has well-conditioned and poorly conditioned eigenvalues [2,7]. Some properties of the Frank matrix $F$ are reported $[2,4]$ as: The eigenvalues of the matrix $F$ are real and positive and also come in reciprocal pair, $\operatorname{det}(F)=1$, the inverse of $F$ is an upper Hessenberg matrix, $L U$ decomposition of $F$ exists, the characteristic polynomial of $F$ has the recurrence relation

$$
\begin{gathered}
\chi_{n}(\lambda)=(1-\lambda) \chi_{n-1}(\lambda)-(n-1) \lambda \chi_{n-2}(\lambda), \\
\chi_{1}(\lambda)=1-\lambda \text { and } \chi_{2}(\lambda)=1-3 \lambda+\lambda^{2} .
\end{gathered}
$$

Also, Varah [7] gave a generalization of the Frank matrix and showed how to compute its eigensystem accurately.

Frank matrix is also a Max matrix. There are many matrices defined on maximum and minimum concepts. Some of them have been mentioned by Kılıç and Arıkan [5]. Also, they have introduced new generalizations of the classical Max and Min matrices and have derived their inverses, $L U$ and Cholesky decompositions and their inverse matrices.

In this paper, we define a new generalization of the Frank matrix and examine its some properties such as determinant and $L U$ decomposition.

Received : 09-01-2020, Accepted : 01-05-2020

This article is a part of doctoral thesis being prepared by Efruz Özlem Mersin. 


\section{Structure of Generalized Frank Matrices}

Consider the real $n$-tuple $a=\left(a_{1}, a_{2}, \ldots, a_{n}\right) \in \mathbb{R}^{n}$. The $n$-tuple $a=\left(a_{1}, a_{2}, \ldots, a_{n}\right)$ corresponds to well-known circulant, Min and Max matrices

$$
\left[\begin{array}{ccccc}
a_{1} & a_{2} & a_{3} & \cdots & a_{n} \\
a_{n} & a_{1} & a_{2} & \cdots & a_{n-1} \\
a_{n-1} & a_{n} & a_{1} & \cdots & a_{n-2} \\
\vdots & \vdots & \vdots & \ddots & \vdots \\
a_{2} & a_{3} & a_{4} & \cdots & a_{1}
\end{array}\right],\left[\begin{array}{ccccc}
a_{1} & a_{1} & a_{1} & \cdots & a_{1} \\
a_{1} & a_{2} & a_{2} & \cdots & a_{2} \\
a_{1} & a_{2} & a_{3} & \cdots & a_{3} \\
\vdots & \vdots & \vdots & \ddots & \vdots \\
a_{1} & a_{2} & a_{3} & \cdots & a_{n}
\end{array}\right] \text { and }\left[\begin{array}{ccccc}
a_{1} & a_{2} & a_{3} & \cdots & a_{n} \\
a_{2} & a_{2} & a_{3} & \cdots & a_{n} \\
a_{3} & a_{3} & a_{3} & \cdots & a_{n} \\
\vdots & \vdots & \vdots & \ddots & \vdots \\
a_{n} & a_{n} & a_{n} & \cdots & a_{n}
\end{array}\right]
$$

respectively. For more information about the above matrices, please refer to $[1,6]$.

Let us define a new matrix which corresponds to the real $n$-tuple $a=\left(a_{1}, a_{2}, \ldots, a_{n}\right)$ as follows:

$$
F_{a}=\left[\begin{array}{ccccccc}
a_{n} & a_{n-1} & 0 & 0 & \cdots & 0 & 0 \\
a_{n-1} & a_{n-1} & a_{n-2} & 0 & \cdots & 0 & 0 \\
a_{n-2} & a_{n-2} & a_{n-2} & a_{n-3} & \cdots & 0 & 0 \\
\vdots & \vdots & \vdots & \vdots & \ddots & \vdots & \vdots \\
a_{2} & a_{2} & a_{2} & a_{2} & \cdots & a_{2} & a_{1} \\
a_{1} & a_{1} & a_{1} & a_{1} & \cdots & a_{1} & a_{1}
\end{array}\right]
$$

The elements of the matrix $F_{a}=\left[\left(f_{a}\right)_{i j}\right]$ is generated by the rule:

$$
\left(f_{a}\right)_{i j}=\left\{\begin{array}{cc}
a_{n+1-\max (i, j)}, & i>j-2 \\
0, & \text { otherwise. }
\end{array}\right.
$$

When we take $a_{i}=i(i=1,2, \ldots, n)$, the matrix $F_{a}$ is reduced to the classical Frank matrix $F$ in (1). So, we call $F_{a}$ as the generalized Frank matrix. From matrix multiplication, it is easily seen that the matrix $F_{a}$ is factored as

$$
F_{a}=\widetilde{I} \Lambda P \widetilde{I}
$$

where

$$
P=\left[\begin{array}{cccc}
1 & 1 & \cdots & 1 \\
0 & 1 & \cdots & 1 \\
\vdots & \vdots & \ddots & \vdots \\
0 & 0 & \cdots & 1
\end{array}\right], \Lambda=\left[\begin{array}{ccccc}
a_{1} & 0 & \cdots & 0 & 0 \\
a_{1} & a_{2}-a_{1} & \cdots & 0 & 0 \\
0 & a_{2} & \ddots & \vdots & \vdots \\
\vdots & \vdots & \ddots & a_{n-1}-a_{n-2} & 0 \\
0 & 0 & \cdots & a_{n-1} & a_{n}-a_{n-1}
\end{array}\right]
$$

and

$$
\tilde{I}=\left[\begin{array}{cccc}
0 & 0 & 0 & 1 \\
0 & 0 & 1 & 0 \\
\vdots & & \vdots & \vdots \\
1 & \cdots & 0 & 0
\end{array}\right]
$$

Hence, we have

$$
F=\left[\begin{array}{cccc}
0 & 0 & 0 & 1 \\
0 & 0 & 1 & 0 \\
\vdots & & \vdots & \vdots \\
1 & \cdots & 0 & 0
\end{array}\right]\left[\begin{array}{ccccc}
1 & 0 & \cdots & 0 & 0 \\
1 & 1 & \cdots & 0 & 0 \\
0 & 2 & \ddots & \vdots & \vdots \\
\vdots & \vdots & \ddots & 1 & 0 \\
0 & 0 & \cdots & n-1 & 1
\end{array}\right]\left[\begin{array}{cccc}
1 & 1 & \cdots & 1 \\
0 & 1 & \cdots & 1 \\
\vdots & \vdots & \ddots & \vdots \\
0 & 0 & \cdots & 1
\end{array}\right]\left[\begin{array}{cccc}
0 & 0 & 0 & 1 \\
0 & 0 & 1 & 0 \\
\vdots & & \vdots & \vdots \\
1 & \cdots & 0 & 0
\end{array}\right]
$$

for the classical Frank matrix.

Let $V_{n}$ be a set of all $n \times n$ generalized Frank matrices as in (3). The following theorem gives us the algebraic structure of $V_{n}$. 
Theorem 2.1. $V_{n}$ is an n-dimensional vector space.

Proof. Let $F_{a}=\left[\left(f_{a}\right)_{i j}\right], F_{b}=\left[\left(f_{b}\right)_{i j}\right] \in V_{n}$ for $a=\left(a_{1}, a_{2}, \ldots, a_{n}\right), b=\left(b_{1}, b_{2}, \ldots, b_{n}\right) \in \mathbb{R}^{n}$ and $\alpha, \beta$ be real numbers. If $D=\alpha F_{a}+\beta F_{b}=\left[d_{i j}\right]$, then by equation (4) we have,

$$
d_{i j}=\alpha\left(f_{a}\right)_{i j}+\beta\left(f_{b}\right)_{i j}=\left\{\begin{array}{cc}
\alpha a_{n+1-\max (i, j)}+\beta b_{n+1-\max (i, j)}, & i>j-2 \\
0, & \text { otherwise. }
\end{array}\right.
$$

Also,

$$
\alpha a+\beta b=\left(\alpha a_{1}+\beta b_{1}, \ldots, \alpha a_{n}+\beta b_{n}\right)=\left(c_{1}, c_{2}, \ldots, c_{n}\right)=c \in \mathbb{R}^{n},
$$

where $c_{i}=\alpha a_{i}+\beta b_{i} \quad(i=1, \ldots, n)$. If $F_{c}=\left[\left(f_{c}\right)_{i j}\right] \in V_{n}$ corresponds to the $n$-tuple $c=\left(c_{1}, c_{2}, \ldots, c_{n}\right)$, then

$$
\begin{aligned}
\left(f_{c}\right)_{i j} & =\left\{\begin{array}{cc}
c_{n+1-\max (i, j)}, & i>j-2 \\
0, & \text { otherwise. }
\end{array}\right. \\
& =\left\{\begin{array}{cc}
\alpha a_{n+1-\max (i, j)}+\beta b_{n+1-\max (i, j)}, & i>j-2 \\
0, & \text { otherwise. }
\end{array}\right.
\end{aligned}
$$

Hence, considering the equations (6), (7) we get

$$
F_{c}=F_{\alpha a+\beta b}=\alpha F_{a}+\beta F_{b} \in V_{n} .
$$

Thus, $V_{n}$ is a subspace of the vector space of all $n \times n$ matrices.

Let $1_{s}$ be an $n$-tuple such that its $s$ th element is one and the others are zero. For example, $1_{3}=(0,0,1,0, \ldots, 0)$. Then for every $F_{a} \in V_{n}$, we have

$$
F_{a}=\sum_{s=1}^{n} a_{s} F_{1_{s}}
$$

and the matrices $F_{1_{1}}, F_{1_{2}}, \ldots, F_{1_{n}}$ are linear independent. Then the $n$ matrices $F_{1_{1}}, F_{1_{2}}, \ldots, F_{1_{n}}$ form a basis for $V_{n}$. That is, the dimension of $V_{n}$ is $n$.

The question is while the matrix $F$ is generalized to the matrix $F_{a}$, how we can generalize the determinant, inverse, $L U$ decomposition and characteristic polynomial formulas given in the study of Hake [4]? In the present paper, we seek answer to this question.

To characterize our results, we use the term $a_{0}$ in this paper. Our readers should know that $a_{0}=0$ throughout this paper.

Next section presents our results.

\section{Main Results}

Theorem 3.1. The determinant of the $n \times n$ matrix $F_{a}$ is

$$
\operatorname{det}\left(F_{a}\right)=\prod_{i=1}^{n}\left(a_{i}-a_{i-1}\right) .
$$

Proof. According to equation (5), we obtain

$$
\operatorname{det}\left(F_{a}\right)=\operatorname{det}(\widetilde{I}) \operatorname{det}(\Lambda) \operatorname{det}(P) \operatorname{det}(\widetilde{I}) .
$$

Since $\operatorname{det}(\widetilde{I})=\mp 1$ and $\operatorname{det}(P)=1$, we get

$$
\begin{aligned}
\operatorname{det}\left(F_{a}\right) & =\operatorname{det}(\Lambda)=a_{1}\left(a_{2}-a_{1}\right)\left(a_{3}-a_{2}\right) \ldots\left(a_{n}-a_{n-1}\right) \\
& =\prod_{i=1}^{n}\left(a_{i}-a_{i-1}\right) .
\end{aligned}
$$


Corollary 3.1. $F_{a}$ is invertible if and only if $a_{i} \neq a_{i-1}$ for $i=1,2, \ldots, n$.

Theorem 3.2. Let the matrix $\left(B_{a}\right)_{n}=\left(\beta_{i j}\right)_{i, j=1}^{n}$ be the inverse of the $n \times n$ matrix $F_{a}$. Then, we have

$$
\beta_{i j}=\left\{\begin{array}{cl}
\frac{1}{a_{n}-a_{n-1}}, & i=j=1 \\
\frac{a_{n+2-i}}{\left(a_{\left.n+2-i-a_{n+1-i}\right)\left(a_{n+1-i}-a_{n-i}\right)},\right.} & i=j \neq 1 \\
-\frac{1}{a_{n+2-i}-a_{n+1-i}}, & i=j+1 \\
0, & i>j+1 \\
(-1)^{j-i} \prod_{k=1}^{j-i} \beta_{i i} \frac{a_{n+1-i-k}}{\left(a_{n+1-i-k}-a_{n-i-k}\right)}, & i<j
\end{array}\right.
$$

where $a_{i} \neq a_{i-1}(i=1,2, \ldots, n)$.

Proof. We use principle of mathematical induction on $n$. It is clear that the result is true for $n=2$, that is,

$$
F_{a}=\left[\begin{array}{ll}
a_{2} & a_{1} \\
a_{1} & a_{1}
\end{array}\right]
$$

and

$$
\begin{aligned}
\left(B_{a}\right)_{2} & =\frac{1}{a_{2} a_{1}-a_{1}^{2}}\left[\begin{array}{cc}
a_{1} & -a_{1} \\
-a_{1} & a_{2}
\end{array}\right]=\left[\begin{array}{cc}
\frac{1}{a_{2}-a_{1}} & \frac{-a_{1}}{\left(a_{2}-a_{1}\right) a_{1}} \\
\frac{-1}{a_{2}-a_{1}} & \frac{a_{2}}{\left(a_{2}-a_{1}\right) a_{1}}
\end{array}\right] \\
& =\left[\begin{array}{cc}
\frac{1}{a_{2}-a_{1}} & \frac{-a_{1}}{\left(a_{2}-a_{1}\right)\left(a_{1}-a_{0}\right)} \\
\frac{-1}{a_{2}-a_{1}} & \frac{a_{2}}{\left(a_{2}-a_{1}\right)\left(a_{1}-a_{0}\right)}
\end{array}\right]
\end{aligned}
$$

Assume that the result is true for $n-1$, then

$$
\left(B_{a}\right)_{n-1}=\left(\beta_{i j}\right)_{i, j=1}^{n-1}=\left\{\begin{array}{cl}
\frac{1}{a_{n-1}-a_{n-2}}, & i=j=1 \\
\frac{a_{n+1-i}}{\left(a_{n+1-i}-a_{n-i}\right)\left(a_{n-i}-a_{n-1-i}\right)}, & i=j \neq 1 \\
-\frac{1}{a_{n+1-i}-a_{n-i}}, & i=j+1 \\
0, & i>j+1 \\
(-1)^{j-i} \prod_{k=1}^{j-i} \beta_{i i} \frac{a_{n-i-k}}{a_{n-i-k}-a_{n-1-i-k}}, & i<j .
\end{array}\right.
$$

Now, we must show that the result is true for $n$. Let the matrices $F_{a}$ and $\left(B_{a}\right)_{n}$ be partitioned as

$$
F_{a}=\left[\begin{array}{ll}
A_{11} & A_{12} \\
A_{21} & A_{22}
\end{array}\right] \text { and }\left(B_{a}\right)_{n}=\left[\begin{array}{ll}
B_{11} & B_{12} \\
B_{21} & B_{22}
\end{array}\right]
$$

where

$$
\begin{gathered}
A_{11}=\left[a_{n}\right], \\
A_{12}=\left[\begin{array}{lccccc}
a_{n-1} & 0 & 0 & 0 & \cdots & 0
\end{array}\right], \\
A_{21}=\left[\begin{array}{llllll}
a_{n-1} & a_{n-2} & a_{n-3} & \cdots & a_{2} & a_{1}
\end{array}\right]^{T}
\end{gathered}
$$

and

$$
A_{22}=\left[\begin{array}{ccccccc}
a_{n-1} & a_{n-2} & 0 & 0 & \cdots & 0 & 0 \\
a_{n-2} & a_{n-2} & a_{n-3} & 0 & \cdots & 0 & 0 \\
a_{n-3} & a_{n-3} & a_{n-3} & a_{n-4} & \cdots & 0 & 0 \\
\vdots & \vdots & \vdots & \vdots & \ddots & \vdots & \vdots \\
a_{2} & a_{2} & a_{2} & a_{2} & \cdots & a_{2} & a_{1} \\
a_{1} & a_{1} & a_{1} & a_{1} & \cdots & a_{1} & a_{1}
\end{array}\right]
$$


Using the assumption, we have $A_{22}^{-1}=\left(B_{a}\right)_{n-1}$. Then, the equation

$$
\left[\begin{array}{ll}
A_{11} & A_{12} \\
A_{21} & A_{22}
\end{array}\right]\left[\begin{array}{ll}
B_{11} & B_{12} \\
B_{21} & B_{22}
\end{array}\right]=\left[\begin{array}{ll}
I & 0 \\
0 & I
\end{array}\right]
$$

yields

$$
\begin{aligned}
& B_{11}=\left(A_{11}-A_{12} A_{22}^{-1} A_{21}\right)^{-1}=\frac{1}{a_{n}-a_{n-1}}, \\
& B_{12}=-B_{11} A_{12} A_{22}^{-1} \\
& =\left[\begin{array}{llll}
-\frac{x_{1} a_{n-1}}{a_{n-1}-a_{n-2}} & \frac{x_{1} a_{n-1} a_{n-2}}{\left(a_{n-1}-a_{n-2}\right)\left(a_{n-2}-a_{n-3}\right)} & \cdots & (-1)^{n-1} x_{1} \prod_{i=1}^{n-1} \frac{a_{i}}{a_{i}-a_{i-1}}
\end{array}\right]
\end{aligned}
$$

where $x_{1}=\frac{1}{a_{n}-a_{n-1}}$,

$$
B_{21}=-A_{22}^{-1} A_{21} B_{11}=\left[\begin{array}{lllll}
-\frac{1}{a_{n}-a_{n-1}} & 0 & 0 & \cdots & 0
\end{array}\right]^{T}
$$

and

$$
\begin{gathered}
B_{22}=A_{22}^{-1}-A_{22}^{-1} A_{21} B_{11} A_{12} A_{22}^{-1} \\
=\left[\begin{array}{ccccc}
x_{2} & -\frac{x_{2} a_{n-2}}{a_{n-2}-a_{n-3}} & \frac{x_{2} a_{n-2} a_{n-3}}{\left(a_{n-2}-a_{n-3}\right)\left(a_{n-3}-a_{n-4}\right)} & \cdots & (-1)^{n-2} x_{2} \prod_{i=1}^{n-2} \frac{a_{i}}{a_{i}-a_{i-1}} \\
-\frac{1}{a_{n-1}-a_{n-2}} & x_{3} & -\frac{x_{3} a_{n-3}}{a_{n-3}-a_{n-4}} & \cdots & (-1)^{n-3} x_{3} \prod_{i=1}^{n-3} \frac{a_{i}}{a_{i}-a_{i-1}} \\
0 & -\frac{1}{a_{n-2}-a_{n-3}} & x_{4} & \cdots & (-1)^{n-4} x_{4} \prod_{i=1}^{n-4} \frac{a_{i}}{a_{i}-a_{i-1}} \\
\vdots & \vdots & \vdots & \ddots & \vdots \\
0 & 0 & 0 & \cdots & x_{n}
\end{array}\right]
\end{gathered}
$$

where $\underset{2 \leq s \leq n}{x_{s}}=\frac{a_{n+2-s}}{\left(a_{n+2-s}-a_{n+1-s}\right)\left(a_{n+1-s}-a_{n-s}\right)}$. Thus,

$$
\left(B_{a}\right)_{n}=\left[\begin{array}{ccccc}
x_{1} & -\frac{x_{1} a_{n-1}}{a_{n-1}-a_{n-2}} & \frac{x_{1} a_{n-1} a_{n-2}}{\left(a_{n-1}-a_{n-2}\right)\left(a_{n-2}-a_{n-3}\right)} & \cdots & (-1)^{n-1} x_{1} \prod_{i=1}^{n-1} \frac{a_{i}}{a_{i}-a_{i-1}} \\
-\frac{1}{a_{n}-a_{n-1}} & x_{2} & -\frac{x_{2} a_{n-2}}{a_{n-2}-a_{n-3}} & \cdots & (-1)^{n-2} x_{2} \prod_{i=1}^{n-2} \frac{a_{i}}{a_{i}-a_{i-1}} \\
0 & -\frac{1}{a_{n-1}-a_{n-2}} & x_{3} & \cdots & (-1)^{n-3} x_{3} \prod_{i=1}^{n-3} \frac{a_{i}}{a_{i}-a_{i-1}} \\
\vdots & \vdots & \vdots & \ddots & \vdots \\
0 & 0 & 0 & \cdots & x_{n}
\end{array}\right] .
$$

Theorem 3.3. The $L U$ decomposition of $F_{a}$ exists for all $n$. Its factors $L=\left(l_{i j}\right)$ and $U=\left(u_{i j}\right)$ are given by

$$
l_{i j}=\left\{\begin{array}{cc}
0, & i<j \\
1, & i=j \\
\frac{a_{n+1-i}}{a_{n+1-j},} & \text { otherwise }
\end{array} \text { and } u_{i j}=\left\{\begin{array}{cc}
a_{n}, & i=j=1 \\
\frac{\left(a_{n+1-i}\right)\left(a_{n+2-i}-a_{n+1-i}\right)}{a_{n+2-i}}, & i=j \neq 1 \\
a_{n-i}, & i=j-1 \\
0, & \text { otherwise. }
\end{array}\right.\right.
$$

Proof. Matrix multiplication yields the result.

Theorem 3.4. The characteristic polynomial of $F_{a}$ satisfies the recurrence relation

$$
\begin{gathered}
P_{n}(\lambda)=\left(\lambda-a_{n}+a_{n-1}\right) P_{n-1}(\lambda)-a_{n-1} \lambda P_{n-2}(\lambda), \\
P_{1}(\lambda)=\lambda-a_{1} \text { and } P_{2}(\lambda)=\lambda^{2}-\left(a_{1}+a_{2}\right) \lambda+a_{1} a_{2}-a_{1}^{2} .
\end{gathered}
$$


Proof. For the characteristic polynomial of $F_{a}$, we have

$$
\begin{aligned}
& P_{n}(\lambda)=\left|\begin{array}{cccccc}
\lambda-a_{n} & -a_{n-1} & 0 & \cdots & 0 & 0 \\
-a_{n-1} & \lambda-a_{n-1} & -a_{n-2} & \cdots & 0 & 0 \\
-a_{n-2} & -a_{n-2} & \lambda-a_{n-2} & \cdots & 0 & 0 \\
\vdots & \vdots & \vdots & \ddots & \vdots & \vdots \\
-a_{2} & -a_{2} & -a_{2} & \cdots & \lambda-a_{2} & -a_{1} \\
-a_{1} & -a_{1} & -a_{1} & \cdots & -a_{1} & \lambda-a_{1}
\end{array}\right| \\
& =\left(\lambda-a_{n}\right)\left|\begin{array}{cccccc}
\lambda-a_{n-1} & -a_{n-2} & 0 & \cdots & 0 & 0 \\
-a_{n-2} & \lambda-a_{n-2} & -a_{n-3} & \cdots & 0 & 0 \\
-a_{n-3} & -a_{n-3} & \lambda-a_{n-3} & \cdots & 0 & 0 \\
\vdots & \vdots & \vdots & \ddots & \vdots & \vdots \\
-a_{2} & -a_{2} & -a_{2} & \cdots & \lambda-a_{2} & -a_{1} \\
-a_{1} & -a_{1} & -a_{1} & \cdots & -a_{1} & \lambda-a_{1}
\end{array}\right| \\
& +\left(a_{n-1}\right)\left|\begin{array}{cccccc}
-a_{n-1} & -a_{n-2} & 0 & \cdots & 0 & 0 \\
-a_{n-2} & \lambda-a_{n-2} & -a_{n-3} & \cdots & 0 & 0 \\
-a_{n-3} & -a_{n-3} & \lambda-a_{n-3} & \cdots & 0 & 0 \\
\vdots & \vdots & \vdots & \ddots & \vdots & \vdots \\
-a_{2} & -a_{2} & -a_{2} & \cdots & \lambda-a_{2} & -a_{1} \\
-a_{1} & -a_{1} & -a_{1} & \cdots & -a_{1} & \lambda-a_{1}
\end{array}\right|
\end{aligned}
$$

The first determinant of the right hand side of the last equality corresponds to the $P_{n-1}(\lambda)$. Let $q(\lambda)$ denotes the second determinant of the right hand side of the last equality. Then,

$$
q(\lambda)=\mid \begin{array}{cccccc|c}
\lambda-a_{n-1} & -a_{n-2} & 0 & \cdots & 0 \\
-a_{n-2} & \lambda-a_{n-2} & -a_{n-3} & \cdots & 0 \\
-a_{n-3} & -a_{n-3} & \lambda-a_{n-3} & \cdots & 0 & \\
\vdots & \vdots & \vdots & & \vdots \\
-a_{2} & -a_{2} & -a_{2} & \cdots & -a_{1} & \\
-a_{1} & -a_{1} & -a_{1} & \cdots & \lambda-a_{1} & \\
& -\left|\begin{array}{cccccc}
\lambda & -a_{n-2} & 0 & \cdots & 0 & 0 \\
0 & \lambda-a_{n-2} & -a_{n-3} & \cdots & 0 & 0 \\
0 & -a_{n-3} & \lambda-a_{n-3} & \cdots & 0 & 0 \\
\vdots & \vdots & \vdots & \ddots & \vdots & \vdots \\
0 & -a_{2} & -a_{2} & \cdots & \lambda-a_{2} & -a_{1} \\
0 & -a_{1} & -a_{1} & \cdots & -a_{1} & \lambda-a_{1}
\end{array}\right| \\
=P_{n-1}(\lambda)-\lambda P_{n-2}(\lambda) . & & &
\end{array}
$$

Thus, we have

$$
\begin{aligned}
P_{n}(\lambda) & =\left(\lambda-a_{n}\right) P_{n-1}(\lambda)+a_{n-1}\left(P_{n-1}(\lambda)-\lambda P_{n-2}(\lambda)\right) \\
& =\left(\lambda-a_{n}+a_{n-1}\right) P_{n-1}(\lambda)-a_{n-1} \lambda P_{n-2}(\lambda) .
\end{aligned}
$$

Also, it is clear that $P_{1}(\lambda)=\lambda-a_{1}$ and $P_{2}(\lambda)=\lambda^{2}-\left(a_{1}+a_{2}\right) \lambda+a_{1} a_{2}-a_{1}^{2}$.

Theorem 3.5. Let $P_{n}(\lambda)=\lambda^{n}+\gamma_{n-1}^{(n)} \lambda^{n-1}+\cdots+\gamma_{1}^{(n)} \lambda+\gamma_{0}^{(n)}$ be the characteristic polynomial of the $n \times n$ matrix $F_{a}$. Then,

and

$$
\begin{gathered}
\gamma_{0}^{(n)}=\left(a_{n-1}-a_{n}\right) \gamma_{0}^{(n-1)}=(-1)^{n} \operatorname{det}\left(F_{a}\right), \\
\gamma_{n-1}^{(n)}=\gamma_{n-2}^{(n-1)}-a_{n}=-\operatorname{tr}\left(F_{a}\right)
\end{gathered}
$$

are valid for $1 \leq i \leq n-2$.

$$
\gamma_{i}^{(n)}=\gamma_{i-1}^{(n-1)}+\left(a_{n-1}-a_{n}\right) \gamma_{i}^{(n-1)}-a_{n-1} \gamma_{i-1}^{(n-2)}
$$


Proof. By using the recurrence relation in Theorem 3.4 and the coefficients of $P_{n}(\lambda), P_{n-1}(\lambda)$ and $P_{n-2}(\lambda)$, we have

$$
\begin{array}{r}
\lambda^{n}+\gamma_{n-1}^{(n)} \lambda^{n-1}+\cdots+\gamma_{1}^{(n)} \lambda+\gamma_{0}^{(n)}=\left(\lambda-a_{n}+a_{n-1}\right)\left(\lambda^{n-1}+\gamma_{n-2}^{(n-1)} \lambda^{n-2}+\cdots+\gamma_{1}^{(n-1)} \lambda+\gamma_{0}^{(n-1)}\right) \\
-a_{n-1} \lambda\left(\lambda^{n-2}+\gamma_{n-3}^{(n-2)} \lambda^{n-3}+\cdots+\gamma_{1}^{(n-2)} \lambda+\gamma_{0}^{(n-2)}\right) .
\end{array}
$$

Comparison of the coefficients yields the desired formulas. Also, we have

$$
\begin{aligned}
\gamma_{0}^{(n)} & =\left(a_{n-1}-a_{n}\right) \gamma_{0}^{(n-1)}=\left(a_{n-1}-a_{n}\right)\left(a_{n-2}-a_{n-1}\right) \gamma_{0}^{(n-2)} \\
& =\cdots=(-1)^{n} \prod_{i=1}^{n}\left(a_{i}-a_{i-1}\right)=(-1)^{n} \operatorname{det}\left(F_{a}\right)
\end{aligned}
$$

and

$$
\gamma_{n-1}^{(n)}=\gamma_{n-2}^{(n-1)}-a_{n}=\gamma_{n-3}^{(n-2)}-a_{n-1}-a_{n}=\cdots=-\left(a_{1}+a_{2}+\cdots+a_{n}\right)=-\operatorname{tr}\left(F_{a}\right) .
$$

\section{Conclusion}

In this paper we introduced a new generalized Frank matrix. Then, we examined its algebraic structure, determinant, inverse, $L U$ decomposition and characteristic polynomial. We showed that the determinant, inverse, $L U$ decomposition and characteristic polynomial formulas of the generalized Frank matrix are the general forms those of classical Frank matrix. When we take $a_{i}=i(i=1,2, \ldots, n)$, our results are reduced the results of Hake [4].

\section{References}

[1] Davis, P. J.: Circulant Matrices. Wiley, New York. Chichester, Brisbane (1979).

[2] Eberlein, P. J.: A note on the matrices denoted $B_{n}^{*}$. Siam J. Appl. Math. 20, 87-92 (1971). https://doi.org/10.1137/0120012

[3] Frank, W.L.: Computing eigenvalues of complex matrices by determinant evaluation and by methods of Danilewski and Wielandt. J. Soc. Indust. Appl. Math. 6 (4), 378-392 (1958). https:/ / doi.org/10.1137/0106026

[4] Hake, J-F.: A remark on Frank matrices. Computing. 35, 375-379 (1985). https:/ / doi.org/10.1007/BF02240202

[5] Kılıç, E. and Arıkan, T.: Studying new generalizations of Max-Min matrices with a novel approach. Turkish Journal of Mathematics. 43, 2010-2024 (2019). https://doi:10.3906/mat-1811-95

[6] Mattila, M. and Haukkanen, P.: Studying the various properties of Min and Max matrices - elementary vs. more advanced methods. Spec. Matrices. 4, 101-109 (2016). https:/ / doi.org/10.1515/spma-2016-0010

[7] Varah, J.M.: A generalization of the Frank matrix. Siam J.Sci. Stat. Comput. 7(3), 835-839 (1986). https://doi.org/10.1137/0907056

\section{Affiliations}

\section{EFRUZ ÖZLEM MERSIN}

ADDRESS: Aksaray University, Department of Mathematics, 68000, Aksaray-Turkey.

E-MAIL: efruzmersin@gmail.com

ORCID ID:0000-0001-6260-9063

Mustafa BAHŞI

AdDress: Aksaray University, Department of Mathematics and Science Education, 68000, Aksaray-Turkey.

E-MAIL: mhvbahsi@yahoo.com

ORCID ID:0000-0002-6356-6592 
AYŞE DiLeK MADEN

ADDRESS: Selçuk University, Department of Mathematics, 42130, Konya-Turkey. E-MAIL: aysedilekmaden@selcuk.edu.tr.

ORCID ID:0000-0001-7717-0241 